Vol. 42 (1990) [201-213]

\title{
THE DIFFERENTIABILITY OF CONVEX FUNCTIONS ON TOPOLOGICAL LINEAR SPACES
}

\author{
Bernice ShaRP
}

In this paper topological linear spaces are categorised according to the differentiability properties of their continuous convex functions. Mazur's Theorem for Banach spaces is generalised: all separable Baire topological linear spaces are weak Asplund. A class of spaces is given for which Gateaux and Fréchet differentiability of a continuous convex function coincide, which with Mazur's theorem, implies that all Montel Fréchet spaces are Asplund spaces. The effect of weakening the topology of a given space is studied in terms of the space's classification. Any topological linear space with its weak topology is an Asplund space; at the opposite end of the topological spectrum, an example is given of the inductive limit of Asplund spaces which is not even a Gateaux differentiability space.

\section{INTRODUCTION}

This paper synthesises two areas of research: the differentiability of functions on topological linear spaces, and the differentiability of convex functions on Banach spaces.

The study of differentiability properties of convex functions on normed linear spaces essentially began with Mazur in 1933 [11]. Asplund, in his innovative paper of 1968, [1], categorised Banach spaces according to the differentiability properties of their real valued continuous convex functions. He nominated two classes: "strong differentiability space", now called Asplund space, and "weak differentiability space" or weak Asplund space, for a Banach space, $X$, with the property that every continuous convex function is Fréchet, or in the weak case, Gateaux, differentiable on a dense $G_{\delta}$ subset of its open convex domain in $X$. In this paper, similar classifications are given, and theorems proved, for topological linear spaces. All spaces are Hausdorff.

\section{Preliminaries}

For a topological linear space $X$ and an open convex subset $D$ of $X$, a real valued function $f$ on $D$ is said to be convex whenever for all $x, y \in D$ and for all $\lambda \in[0,1]$,

$$
f(\lambda x+(1-\lambda) y) \leqslant \lambda f(x)+(1-\lambda) f(y)
$$

\footnotetext{
Received 19th October, 1989.
}

This work was completed as part of a doctoral thesis submitted to the University of Newcastle, NSW, under the supervision of Prof. J.R.Giles.

Copyright Clearance Centre, Inc. Serial-fee code: 0004-9729/90 \$A2.00+0.00. 
and locally Lipschitz whenever for all $\varepsilon>0$, there exists a neighbourhood $U$ of 0 in $X$ such that for all $\alpha \in[0,1]$ and for all $u, v \in(x+U) \cap D$,

$$
u-v \in \alpha U \quad \text { implies } \quad|f(u)-f(v)|<\alpha \varepsilon .
$$

If $X$ is a normed space, the latter definition coincides with the standard one. Continuous convex functions on a topological linear space are locally Lipschitz ([4], 2.4).

The continuous dual of a topological linear space $X$ is denoted by $X^{*}$. Let $U$ be an open subset of $X$ and $\mathcal{M}$ be a bornology on $X$, that is, a class of bounded subsets containing all singletons. A real valued function $f$ on $U$ is $\mathcal{M}$-differentiable at $x \in U$ whenever there exists $u \in X^{*}$ such that, for all $M \in \mathcal{M}$, for all $\varepsilon>0$, there exists $\delta>0$, such that for all $y \in M$, for all $t: 0<|t|<\delta$,

$$
\left|\frac{f(x+t y)-f(x)}{t}-u(y)\right|<\varepsilon .
$$

The map $u: U \rightarrow \mathbf{R}$ is uniquely determined by $f$ and $x$ and is denoted by $f^{\prime}(x)$.

If $\mathcal{M}$ is the class of all bounded subsets of $X$ then $f$ is Fréchet differentiable at $x$. This is the weakest of all possible choices for the derivative which coincide, when $X$ is a normed space, with the standard Fréchet derivative. If $\mathcal{M}$ is the class of all compact subsets of $X$ then $f$ is Hadamard differentiable at $x$; if $\mathcal{M}$ is the class of all singleton subsets of $X$ then $f$ is Gateaux differentiable at $x$.

It is not difficult to show that a continuous convex function $f$ is Gateaux differentiable at $x \in X$ if and only if the function $\phi: X \rightarrow \mathbf{R}$ defined by

$$
\phi(y)=\lim _{t \rightarrow 0} \frac{f(x+t y)-f(x)}{t}
$$

exists for all $y \in X$, that is, $\phi$ is linear and continuous.

In 3.1 we will see that for continuous convex functions, Hadamard and Gateaux differentiability coincide, so in the sequel "Hadamard" could often replace "Gateaux"; however we will use the latter to more obviously compare our results with those known for Banach spaces. Clearly the above derivatives are given in decreasing order of strength; in general the three are distinct (see [2], p.102 and [3], p.209).

\section{The Classification of Differentiability Spaces}

A topological linear space will be classified, using the following abbreviations, according to the differentiability properties of the specified class of real valued convex functions. (A generic set in $D$ is one which contains a dense $G_{\delta}$ subset of $D$.) 
ASP (WASP): Asplund (Weak Asplund): every continuous convex function with domain a nonempty open convex subset is Fréchet (Gateaux) differentiable on a generic set in that domain.

FDS (GDS): Fréchet (Gateaux) Differentiability Space: every continuous convex function with domain a nonempty open convex subset is Fréchet (Gateaux) differentiable on a dense subset of that domain.

These classifications are used in the literature for Banach spaces. In some special cases the four are not distinct, for example for Banach spaces it is well known that the points of Fréchet differentiability of any continuous convex function form a $G_{6}$ set in its domain, so in this case ASP and FDS are equivalent.

\section{A Generalisation of MazUr's Theorem}

Theorem 2.1 is a generalisation of Mazur's Theorem, which states that every separable Banach space is WASP. This proof follows some of the ideas incorporated in the original [11].

Theorem 2.1. All separable Baire topological linear spaces are WASP.

Proof: Let $X$ be a separable Baire topological linear space and let $f$ be a continuous convex function on a nonempty open convex subset $D$ of $X$. Let $\left\{x_{n}: n \in N\right\}$ be a countable dense subset of $D$; denote by $M$ the set of points in $D$ where $f$ is Gateaux differentiable.

For each $n \in N$, define $M_{n}$ by

$$
M_{n}=\left\{x \in D: \lim _{t \rightarrow 0} \frac{f\left(x+t x_{n}\right)-f(x)}{t} \quad \text { exists }\right\} .
$$

It is immediate from the definitions that, for all $n \in \mathrm{N}, M \subset M_{n}$.

Conversely, if $a \in \bigcap_{n=1}^{\infty} M_{n}$ then for all $n \in N, f_{+}^{\prime}(a)\left(x_{n}\right)=f_{-}^{\prime}(a)\left(x_{n}\right)$. Since $f$ is continuous at $a$, it is an easy consequence of the convexity of $f$ that $f_{+}^{\prime}(a)$ and $f_{-}^{\prime}(a)$ are continuous on $X([15], 2.3 .5)$, so for all $x \in X, f_{+}^{\prime}(a)(x)=f_{-}^{\prime}(a)(x)$; thus $a \in M$. Hence $M=\bigcap_{n=1}^{\infty} M_{n}$.

For positive integers $n, i, j$, define $M_{n, i, j}$ by

$$
\begin{aligned}
M_{n, i, j}= & \left\{x \in D: \text { for all } t \in\left(0, \frac{1}{j}\right],\right. \\
& \left.\frac{f\left(x+t x_{n}\right)-f(x)}{t}-\frac{f(x)-f\left(x-t x_{n}\right)}{t} \geqslant \frac{1}{i}\right\} .
\end{aligned}
$$

These sets are closed in $D$ and $D \backslash M_{n}=\bigcup_{i, j=1}^{\infty} M_{n, i, j}$, so each $M_{n}$ is $G_{6}$ in $D$. 
Since $X$ is a Baire space, $M$ is a dense $G_{\delta}$ subset if every $M_{n}$ is a dense $G_{\delta}$ subset. It remains to show that each $M_{n}$ is dense in $D$. Suppose $a \in D$ and $U$ is a balanced neighbourhood of 0 such that $a+U \subset D$. Then for each $n$ there exists $\delta>0$ such that for all $\lambda \in(-\delta, \delta), \lambda x_{n} \in U$. Let $I=(-\delta, \delta)$ and define $g: I \rightarrow \mathrm{R}$ by

$$
g(\lambda)=f\left(a+\lambda x_{n}\right) \text {. }
$$

Since $g$ is continuous and convex, and R is WASP, there exists $\lambda_{0} \in I$ such that $g$ is Gateaux differentiable at $\lambda_{0}$; it follows that $a+\lambda_{0} x_{n} \in M_{n}$, which confirms the density of $M_{n}$ in $U$.

All complete metric spaces are Baire, so, for example, separable Fréchet spaces are WASP. It is well known that "separable" is not a necessary condition in 2.1: all weakly compactly generated Banach spaces are WASP ([1], Theorem 2).

\section{EXAMPLES 2.2 .}

(1) Denote by $C(\mathbf{R})$ the set of real valued continuous functions on $\mathbf{R}$ with topology defined by the set of seminorms $\left\{p_{n}: n=1,2,3 \ldots\right\}$, where

$$
p_{n}(x)=\sup _{t \in[-n, n]}|x(t)| \text {. }
$$

This space is separable and Baire, hence WASP, and provides a generalisation of [10], problem 10.

(2) Let $C(X)$ be the set of real valued functions continuous on the topological space $X$ with the topology of compact convergence on $X$, that is, the topology defined by the family of seminorms

$$
p_{A}(x)=\sup _{t \in A}|x(t)|
$$

where $A$ ranges over all compact subsets of $X$. From 2.1, $C(X)$ is WASP whenever Baire and separable. (For $C(X)$ to be separable it is sufficient that $X$ be $\sigma$-compact and metrisable).

(3) $C^{\infty}(\mathbf{R})$, the space of indefinitely differentiable functions with the topology defined by the set

$$
\left\{p_{m, n}: m=0,1, \ldots ; n=1,2, \ldots\right\}
$$

of seminorms, where

$$
p_{m, n}(x)=\sup _{t \in[-n, n]}\left|x^{(m)}(t)\right|,
$$

is a Montel Fréchet space, hence is Baire and separable ([9], p.370) so is WASP.

(4) Let $D$ be an open subset of the complex plane $C$ and denote by $\mathcal{H}(D)$ the set of all holomorphic functions from $D$ into $C$. This is a subspace of $C(D)$; consider $\mathcal{H}(D)$ with the induced topology of compact convergence. Then $\mathcal{H}(D)$ is also a Montel Fréchet space and is WASP.

It is shown later, in 3.5 , that both $C^{\infty}(\mathbf{R})$ and $\mathcal{H}(D)$ are ASP. 


\section{The Coincidence of Fréchet and Gateaux Differentiability}

In this section it is shown that for locally Lipschitz functions, and hence for continuous convex functions, on a topological linear space, Hadamard and Gateaux differentiability coincide, that is, pointwise convergence of the difference quotient implies convergence uniform on compact subsets of $X$.

Under what conditions do Fréchet and Hadamard differentiability coincide? We need to look at uniform convergence on compact, and on bounded, subsets, so the sufficiency condition that bounded subsets be relatively compact, is intuitively suggested by the definitions. In normed linear spaces this is of little interest, since such spaces are finite dimensional. However a large class of non-normable locally convex spaces has this property.

ThEOREM 3.1. Let $X$ be a topological linear space, and let $f$ be a real valued locally Lipschitz function on a nonempty open subset $D$ of $X$. Hadamard and Gateaux differentiability coincide for $f$.

Proof: To see the nontrivial direction, let $M$ be a compact set in $X$; assume that $f$ is locally Lipschitz, and Gateaux differentiable at $x_{0} \in D$.

Let $y \in D$ and choose $\varepsilon>0$; then the following property holds. There is an open neighbourhood $U_{y}$ of 0 , and a $\delta_{y}>0$ such that for all $z \in y+U_{y}$, for all $t: 0<|t|<\delta_{y}$,

$$
\left|\frac{f\left(x_{0}+t z\right)-f\left(x_{0}\right)}{t}-f^{\prime}\left(x_{0}\right)(z)\right|<\varepsilon .
$$

To see this, define

$$
F_{t}(y)=\frac{f\left(x_{0}+t y\right)-f\left(x_{0}\right)}{t}
$$

If $z \in D$,

$$
\begin{aligned}
\left|F_{t}(z)-f^{\prime}\left(x_{0}\right)(z)\right| \leqslant & \left|F_{t}(z)-F_{t}(y)\right| \\
& +\left|F_{t}(y)-f^{\prime}\left(x_{0}\right)(y)\right| \\
& +\left|f^{\prime}\left(x_{0}\right)(y)-f^{\prime}\left(x_{0}\right)(z)\right| \\
= & A_{1}+A_{2}+A_{3} .
\end{aligned}
$$

For $A_{1}$,

$$
\left|F_{t}(z)-F_{t}(y)\right|=\left|\frac{f\left(x_{0}+t z\right)-f\left(x_{0}+t y\right)}{t}\right|,
$$

and since $f$ is locally Lipschitz, there is a neighbourhood $U_{1}$ of 0 and a $\delta_{1}>0$ such that for all $z \in y+U_{1}$ for all $t: 0<|t|<\delta_{1}$,

$$
\left|\frac{f\left(x_{0}+t y\right)-f\left(x_{0}+t z\right)}{t}\right|<\frac{\varepsilon}{3} \text {. }
$$


For $A_{2}$, since $f$ is Gateaux differentiable at $x_{0}$ there is a $\delta_{2}>0$ such that for all $t: 0<|t|<\delta_{2}$,

$$
\left|F_{t}(y)-f^{\prime}\left(x_{0}\right)(y)\right|<\frac{\varepsilon}{3} .
$$

For $A_{3}$, since $f^{\prime}\left(x_{0}\right)$ is linear, $\left|f^{\prime}\left(x_{0}\right)(y)-f^{\prime}\left(x_{0}\right)(z)\right|=\left|f^{\prime}\left(x_{0}\right)(y-z)\right|$; by continuity there is a neighbourhood $U_{3}$ of 0 such that for all $z \in y+U_{3}$,

$$
\left|f^{\prime}\left(x_{0}\right)(y)-f^{\prime}\left(x_{0}\right)(z)\right|<\frac{\varepsilon}{3} .
$$

Choose $\delta_{y}=\min \left\{\delta_{1}, \delta_{2}\right\}$ and $U_{y}=U_{1} \cap U_{3}$, to confirm (*).

To establish convergence uniformly on $M$, for each $y \in M$, choose an open neighbourhood $U_{y}$ of $y$ and $\delta_{y}>0$ satisfying (*). The family $U=\left\{U_{y}: y \in M\right\}$ is an open covering of $M$, and since $M$ is compact, a finite subset of $\mathcal{U}$, say $\left\{U_{y_{1}}, U_{y_{2}}, \ldots U_{y_{m}}\right\}$, covers $M$; let $\delta=\min \left\{\delta_{y_{i}}: i=1,2, \ldots, m\right\}$.

For all $y \in M$, for all $t: 0<|t|<\delta$,

$$
\left|\frac{f\left(x_{0}+t z\right)-f\left(x_{0}\right)}{t}-f^{\prime}\left(x_{0}\right)(z)\right|<\varepsilon,
$$

which proves that $f$ is Hadamard differentiable at $x_{0}$.

THEOREM 3.2. Let $X$ be a topological linear space in which all bounded subsets are relatively compact and $f$ a real valued function on a nonempty open subset $D$ of $X$. Fréchet and Hadamard differentiability coincide for $f$.

Proof: Suppose that $M$ is a bounded set in $X$ and that $f$ is Hadamard differentiable at $x_{0} \in D$. Since the closure of a bounded set is bounded, we will assume without loss of generality that $M$ is closed. Then by hypothesis $M$ is compact, so we have uniform convergence of the difference quotient on $M$ as required.

Theorem 3.3 is a consequence of Theorems 3.1 and 3.2.

THEOREM 3.3. If $X$ is a topological linear space in which bounded subsets are relatively compact, and if $f$ is a real valued locally Lipschitz function on a nonempty open subset $D$ of $X$, then for $f$, Fréchet and Gateaux differentiability coincide.

Since continuous convex functions are locally Lipschitz, a continuous convex function defined on a nonempty open convex subset of a space in which bounded subsets are relatively compact, Fréchet and Gateaux differentiability coincide.

Theorem 3.4. Montel Fréchet spaces are ASP.

Proof: Fréchet spaces are Baire and Montel Fréchet spaces are separable ([9], p.370), so the conclusion follows from 2.1 and 3.3. 
EXamples 3.5. The spaces $C^{\infty}(\mathrm{R})$ and $\mathcal{H}(D)$ defined in 2.2 are Montel Fréchet spaces and so are ASP.

Finite dimensional spaces. A normed space has bounded sets relatively compact if and only if it is finite dimensional; so Fréchet and Gateaux differentiability coincide for locally Lipschitz functions on finite dimensional normed spaces. This, combined with the generalisation of Mazur's Theorem, 2.1, provides another proof of the well known fact that finite dimensional normed linear spaces are ASP.

\section{Varying the Topology}

It is well known that differentiability properties of real valued functions on a normed linear space are invariant for equivalent norms, that is, these properties depend only on the topology. For a general topological linear space, it is relevant to ask how a change of topology affects the classification of the space. The answer is more dramatic than one might anticipate!

First we show how some differentiability properties relative to one topology are retained relative to any weaker topology. It is easier to prove that a space is GDS or FDS than WASP or ASP, since a $G_{6}$ set in the given topology is not necessarily $G_{6}$ in a weaker topology.

THEOREM 4.1. Suppose that $\mathcal{T}_{1}, \mathcal{T}_{2}$ are linear topologies on a linear space $X$ such that $\mathcal{T}_{1}$ is at least as strong as $\mathcal{T}_{2}$. Whenever $\left(X, \mathcal{T}_{1}\right)$ is GDS, so is $\left(X, \mathcal{T}_{2}\right)$.

Proof: Let $f: X \rightarrow R$ be convex and $T_{2}$-continuous on a $\mathcal{T}_{2}$-open convex subset $U$ of $X$. Then $U$ is $T_{1}$-open and $f$ is $T_{1}$-continuous on $U$, so there is a $\mathcal{T}_{1}$-dense subset $D_{f}$ of $U$, on which $f$ is $T_{1}$-Gateaux differentiable; and $D_{f}$ is $T_{2}$-dense in $U$. Since $f$ is $T_{1}$-Gateaux differentiable on $D_{f}$, it remains only to show that $f^{\prime}\left(x_{0}\right)$ is $\mathcal{T}_{2}$-continuous, but this is an easy consequence of the convexity of $f$ and the fact that $f$ is $T_{2}$-continuous at $x_{0}$.

THEOREM 4.2. Suppose that $\mathcal{T}_{1}$ and $\mathcal{T}_{2}$ are again linear topologies on the linear space $X$ such that $T_{1}$ is at least as strong as $T_{2}$ and further that the $\mathcal{T}_{1}$ - and $\mathcal{T}_{2}$ bounded sets coincide. Whenever $\left(X, \mathcal{T}_{1}\right)$ is FDS, so is $\left(X, \mathcal{T}_{2}\right)$.

Proof: Suppose $f: X \rightarrow \mathbf{R}$ is convex and $\mathcal{T}_{2}$-continuous on a $\mathcal{T}_{2}$-open convex subset $U$. Then $U$ is $\mathcal{T}_{1}$-open, $f$ is $\mathcal{T}_{1}$-continuous and is $\mathcal{T}_{1}$-Fréchet differentiable on a $T_{1}$-dense, hence $T_{2}$-dense, set $D_{f}$ in $U$. Since $f$ is $T_{1}$-Gateaux differentiable on $D_{f}$, the proof of Theorem 4.1 assures us that at each $x_{0} \in D_{f}$ the derivative is $\mathcal{T}_{2}$-continuous and from the hypothesis that $T_{1}$ - and $T_{2}$-bounded sets coincide we conclude that $f$ is $T_{2}$-Fréchet differentiable on $D_{f}$.

(Recall that the $T_{1}$ - and $T_{2}$ - bounded sets coincide whenever $T_{1}$ and $T_{2}$ generate the same dual.) 


\section{WEAK TOPOLOGIES}

If $A$ is a total subset of the algebraic dual of a linear space $X$, the weak topology on $X, \sigma(X, A)$, is generated by the family of seminorms $Q=\left\{q_{G}\right\}$ defined by

$$
q_{G}(x)=\sup _{x^{*} \in G}\left|\left\langle x^{*}, x\right\rangle\right|
$$

where $G$ ranges over all finite subsets of the linear span of $A$.

Convex functions which are continuous on a topological linear space with a weak topology have unexpectedly strong differentiability properties.

Theorem 5.1. Let $X$ be a linear space and let $A$ be a total subset of the algebraic dual of $X$. Consider $X$ with the weak topology $\sigma(X, A)$, let $p$ be a continuous seminorm on $X$, and let $N$ be the null space of $p$. Then $X / N$ is finite dimensional.

Proof: Since $p$ is continuous and convex, $p$ is locally Lipschitz on $X$, that is, if $x \in X$ there exist an open convex subset $D$ of $X, q_{F} \in Q$ and $k \geqslant 0$ such that, for all $x_{1}, x_{2} \in D$,

$$
\left|p\left(x_{1}\right)-p\left(x_{2}\right)\right| \leqslant k q_{F}\left(x_{1}-x_{2}\right) .
$$

Hence if $q_{F}\left(x_{1}-x_{2}\right)=0$ then $p\left(x_{1}\right)=p\left(x_{2}\right)$.

Let $N_{F}$ be the null space of $q_{F}$; if $x$ is in $N_{F}, q_{F}(x)=0$, hence from $(*) p(x)=0$ and $x \in N$; thus $N_{F} \subset N$.

Since $F$ is a finite subset of the linear span of $A, F$ is of the form

$$
F=\left\{x_{i}^{*} \in A: i \leqslant n\right\}
$$

and it follows that

$$
N_{F}=\left\{x \in X:\left\langle x_{i}^{*}, x\right\rangle=0, i \leqslant n\right\} .
$$

Without loss of generality we will assume that the $x_{i}^{*}$ 's are linearly independent. Hence there exist $x_{i} \in X$ such that $\left\langle x_{i}^{*}, x_{j}\right\rangle=\delta_{i j}([14]$, Chapters 2, 5); consider the map $x \mapsto\left(\left\langle x_{1}^{*}, x\right\rangle,\left\langle x_{2}^{*}, x\right\rangle, \ldots\left\langle x_{n}^{*}, x\right\rangle\right)$ to see that $X / N_{F}$ is finite dimensional. Since $N_{F}$ is a subset of $N, X / N$ is finite dimensional.

In Theorem 5.2 similar methods are used to give an analogous result for any continuous convex function. The following lemma, which is an easy consequence of the convexity inequalities, will be needed.

Lemma. Let $X$ be a linear space, $W$ a linear subspace of $X, U$ a nonempty convex subset of $X, x \in X, f: U \rightarrow \mathbf{R}$ convex and $x+W \subset U$. If $f$ is bounded above on $x+W$ then $f$ is constant on $x+W$. 
Proof: Since $W$ is a linear subspace of $X$, for all $w \in W$, for all $r \in \mathbf{R}, r w \in W$, and $x+W \subset U$ so $x+r w \in U$. Choose $m \in \mathbf{R}$ such that for all $w \in W, f(x+w) \leqslant m$. By convexity of $f$, for all $\alpha \in(0,1)$, for all $u \in W$,

$$
-\alpha(m-f(x)) \leqslant f(x+\alpha u)-f(x) \leqslant \alpha(m-f(x)) .
$$

Let $\alpha=\frac{1}{r}$ and $u=r w$; for all $r>1$, for all $w \in W$,

$$
-(m-f(x)) \leqslant r(f(x+w)-f(x)) \leqslant(m-f(x)) .
$$

If $f(x+w)-f(x) \neq 0$, taking $r$ sufficiently large provides a contradiction.

Notation. For the rest of Section $5, X$ is a topological space with the weak topology $\sigma(X, A)$ generated by $A$, a total subset of the algebraic dual of $X$. For each finite linearly independent subset $F$ of $A$, and each $\varepsilon>0$, define $V(F, \varepsilon)$ by

$$
V(F, \varepsilon)=\left\{x \in X:\left|\left\langle x^{*}, x\right\rangle\right|<\varepsilon, x^{*} \in F\right\} .
$$

The $V(F, \varepsilon)$ form a neighbourhood base at the origin. Denote the cardinality of $F$ by $|F|$ and let $\left\{e_{x^{*}}: x^{*} \in F\right\}$ be a basis for $\mathbf{R}^{|F|}$. Define $T_{F}: X \rightarrow \mathbf{R}^{|F|}$ by

$$
T_{F}(x)=\sum_{x^{*} \in F}\left\langle x^{*}, x\right) e_{x^{*}}
$$

The map $T_{F}$ is continuous, open and onto.

THEOREM 5.2. Let $f$ be a continuous real valued convex function on a nonempty open convex subset $U$ of $X$. There is a finite linearly independent subset $F$ of $A$ such that, for each finite linearly independent superset $H$ of $F$, there exists a continuous convex map $f_{H}: T_{H}[U] \rightarrow \mathbf{R}$ for which

$$
f=f_{H} \circ T_{H}
$$

$T_{H}[U]$ is a convex, nonempty, open subset of $R^{|H|}$.

Proof: Let $x_{0} \in U$; since $f$ is continuous at $x_{0}$,

$$
f^{-1}\left[f\left(x_{0}\right)+(-1,1)\right]
$$

is open and contains $x_{0}$, so there exist a finite linearly independent subset $F$ of $A$, and an $\varepsilon>0$, such that

$$
x_{0}+V(F, \varepsilon) \subset\left(f^{-1}\left[f\left(x_{0}\right)+(-1,1)\right] \cap U\right) \subset U .
$$


If $H$ is any finite linearly independent superset of $F$ then

$$
V(H, \varepsilon) \subset V(F, \varepsilon)
$$

so $f$ is bounded on $x_{0}+W$, where $W$ denotes $H^{\perp}$. Let $x \in U$. Since $U$ is open there exists $t>0$ such that

$$
z=(1+t) x-t x_{0} \in U
$$

that is

$$
x=\frac{1}{1+t} z+\frac{t}{1+t} x_{0}
$$

Let $w \in W$ and let $w^{\prime}=\frac{1+t}{t} w$, then $w^{\prime} \in W$ and $x_{0}+w^{\prime} \in U ; w=\frac{t}{1+t} w^{\prime}$, and

$$
\begin{aligned}
x+w & =x+\frac{t}{1+t} w^{\prime} \\
& =\frac{1}{1+t} z+\frac{t}{1+t}\left(x_{0}+w^{\prime}\right)
\end{aligned}
$$

since $U$ is convex, $x+w \in U$. Hence $x+W \subset U$.

Also,

$$
\begin{aligned}
f(x+w) & \leqslant f\left(\frac{1}{1+t} z+\frac{t}{1+t}\left(x_{0}+w^{\prime}\right)\right) \\
& \leqslant \frac{1}{1+t} f(z)+\frac{t}{1+t} f\left(x_{0}+w^{\prime}\right) \\
& \leqslant \frac{1}{1+t} f(z)+\frac{t}{1+t} M
\end{aligned}
$$

where $M=f\left(x_{0}\right)+1$, so $f$ is bounded on $x+W$.

From the preceding lemma, since $f$ is continuous and convex, and bounded on $x+W \subset U, f$ is constant on $x+W$, that is for all $w \in W$,

$$
f(x+w)=f(x)
$$

If $X / W$ is identified with $\mathbf{R}^{|H|}$ then $T_{H}(x)=x+W, f_{H}: U / W \rightarrow \mathbf{R}$ defined by $f_{H}(x+W)=f(x)$ is a well defined function and

$$
f(x)=\left(f_{H} \circ T_{H}\right)(x)
$$

further, $f_{H}$ is continuous (since $T_{H}$ is open, continuous and onto) and convex. 
REMARK 5.3. Using the notation of 5.2, $f$ is Gateaux differentiable at $x \in U$ if and only if $f_{H}$ is Gateaux differentiable at $T_{H}(x)$; if $f_{H}$ is Fréchet differentiable at $T_{H}(x)$ then $f$ is Fréchet differentiable at $x$.

The reverse implication for Fréchet differentiability requires additional conditions. For example it suffices that $T_{H}$ is bound covering, that is, for each bounded set $B$ in $\mathrm{R}^{|H|}$ there exists a bounded set $M$ in $X$ such that $T_{H}[M] \supset B$. (This concept was suggested to me by Prof I Namioka.)

THEOREM 5.4. In a topological linear space with a weak topology, Gateaux and Fréchet differentiability coincide for continuous convex functions. (This was shown to me by Dr. R. Eyland.)

Proof: Using the same notation, let $f$ be a continuous convex function on $U$ and let $x \in U$ be a Gateaux differentiability point of $f$. By 5.2 there exist $F$ and $f_{F}$ such that $f=f_{F} \circ T_{F}$. From 5.3, $T_{F}(x)$ is a Gateaux differentiability point of $f_{F}$; since $R^{|F|}$ is finite dimensional, from 3.6 $T_{F}(x)$ is a Fréchet differentiability point of $f_{F}$. Using 5.3 again, $x$ is a Fréchet differentiability point of $f$.

For the dual of a Banach space with the weak* topology, and for a reflexive Banach space with the weak topology, Theorem 5.4 can also be seen as a consequence of Theorem 3.3.

THEOREM 5.5. Any topological linear space $X$ with a weak topology is ASP.

Proof: It suffices to show that $X$ is WASP (5.4). Again using the same notation, let $f$ be a continuous convex function on $U$. From 5.2 there exists a finite linearly independent subset $F$ of $A$ such that $f=f_{F} \circ T_{F}$. Since $\mathbf{R}^{|F|}$ is WASP, $f_{F}$ is Gateaux differentiable on a dense $G_{\sigma}$ subset $G$ of $T_{F}[U]$. It follows from 5.3 that the set of Gateaux differentiability points of $f$ is $T_{F}^{-1}[G]$ and the continuity of $T_{F}$ guarantees that this set is a $G_{\delta}$ subset of $U$.

It remains to show that $T_{F}^{-1}[G]$ is dense in $U$. Let $x \in U$ and let $N_{x}$ be a neighbourhood of $x$ in $X$. There exist a finite linearly independent subset $H$ of $A$, and an $\varepsilon>0$, such that

$$
x+V(H, \varepsilon) \subset N_{x} .
$$

Without loss of generality, assume that $F \subset B$. Denote by $B(\varepsilon)$ the " $\varepsilon$-box" round $T_{H}(x)$ in $R^{|H|}$, that is,

$$
B(\varepsilon)=T_{H}(x)+\left\{\sum_{x^{*} \in H} \alpha_{x^{*}} e_{x^{*}}:\left|\alpha_{x^{*}}\right|<\varepsilon\right\}
$$

From 5.2, $f=f_{H} \circ T_{H}$, and $f_{H}$ is Gateaux differentiable on a dense subset, say $G_{H}$, of $T_{H}[U]$, so $G_{H} \cap B(\varepsilon)$ is nonempty. 
The set $T_{H}^{-1}\left[G_{H} \cap B(\varepsilon)\right]=T_{H}^{-1}\left[G_{H}\right] \cap T_{H}^{-1}[B(\varepsilon)]$ is nonempty. Using $5.3, T_{H}^{-1}\left[G_{H}\right]$ is the set of Gateaux differentiability points of $f$, so $T_{H}^{-1}\left[G_{H}\right]=T_{F}^{-1}[G]$. Since $T_{H}^{-1}[B(\varepsilon)]=x+V(H, \varepsilon), N_{x}$ contains a point of $T_{F}^{-1}[G]$.

EXAMPLES 5.6. Any Banach space with its weak topology is ASP; the dual of a Banach space with the weak or weak ${ }^{*}$ topology is ASP. The space $\ell_{1}$ with its norm topology is not FDS $([13], 1.14)$; with its weak topology, or, when considered as the dual of $c_{0}$, its weak ${ }^{*}$ topology, $\ell_{1}$ is ASP. Perhaps the most striking example is $\ell_{\infty}$, which with its norm topology is not even GDS $([13], 1.21)$, but with its weak or weak* topology is ASP.

It is an interesting question which convex functions are continuous on a Banach space with its weak topology: Theorem $\mathbf{5 . 2}$ shows that such functions factor through $\mathbf{R}^{n}$, for some $n$.

\section{The Inductive Limit Topology}

Consider a family of locally convex spaces, $\left\{X_{\gamma}: \gamma \in \Gamma\right\}$, where for each $\gamma, X_{\gamma}$ is a subspace of a linear space $X$ and such that their union spans $X$. The strongest locally convex topology on $X$ for which each of the injection maps

$$
i_{\gamma}: X_{\gamma} \rightarrow X
$$

is continuous is the inductive limit topology on $X$; a base of neighbourhoods at 0 for this topology is the set of convex subsets $U$ of $X$ such that for each $\gamma, i_{\gamma}^{-1}(U)$ is a neighbourhood of $\mathbf{0}$ in $X_{\boldsymbol{\gamma}}$.

The interesting problem of whether the inductive limit of Asplund Spaces is itself an Asplund space was suggested to me by Dr. S. Yamamuro. From the following example we conclude that the inductive limit of Asplund Spaces need not even be a Gateaux Differentiability Space.

EXAMPLE 6.1. Let $\mathcal{K}(\mathrm{N})$ be the set of real valued functions of finite support on $N$. For each $n \in N$, define $\mathcal{K}_{n}(N)$ by

$$
\mathcal{K}_{n}(N)=\{x \in \mathcal{K}(N): \text { the support of } x \subset\{0,1,2, \ldots n\}\} .
$$

Each $\mathcal{K}_{n}(N)$ is finite dimensional. Consider each $\mathcal{K}_{n}(N)$ with its unique norm topology and $\mathcal{K}(N)$ with the inductive limit topology induced by the family $\left\{\mathcal{K}_{n}(N): n \in N\right\}$.

Each $\mathcal{K}_{n}(N)$ is ASP, from Theorem 3.6. Let $f$ be the $\ell_{1}$ norm on $\mathcal{K}(N)$; $f$ is continuous as a norm on $\ell_{1}$, hence as a function restricted to $\mathcal{K}(N)$ it is a fortiori continuous for this stronger topology, and is Gateaux differentiable at $x=\left(x_{1}, x_{2}, x_{3}, \ldots\right) \in \ell_{1}$, if and only if each coordinate $x_{i}$ is nonzero (this follows using the method of proof of [13] 1.4(b)). Hence $f$ is nowhere Gateaux differentiable on $\mathcal{K}(N)$. 


\section{REFERENCES}

[1] E. Asplund, 'Fréchet differentiability of convex functions', Acta Math 121 (1968), 31-47.

[2] V.I. Averbukh and O.G. Smulyanov, 'The theory of differentiation in linear topological spaces', Russian Math. Surveys 226 (1967), 201-258.

[3] V.I. Averbukh and O.G. Smulyanov, 'The various definitions of the derivative in linear topological spaces', Russian Math. Surveys 234 (1968), 67-113.

[4] J.M. Borwein, 'Continuity and differentiability properties of convex operators', Proc. London Math. Soc. 3 (1982), 420-444.

[5] J.M. Borwein, 'Generic differentiability of order-bounded convex operators', J. Austral. Math. Soc. Ser. B 28 (1986), 22-29.

[6] M. Coban and P. Kenderov, 'Dense Gateaux differentiability of the sup-norm in $C(T)$ and the topological properties of $T^{\prime}$, C.R.Acad. Bulgare Sci. 38 (1985), 1603-1604.

[7] J.R. Giles, Convex analysis with application in differentiation of convex functions: Research Notes in Mathematics 58 (Pitman, 1982).

[8] J.L. Kelley and I. Namioka, Linear topological spaces (Van Nostrand, Princeton, N.J., 1963).

[9] G. Köthe, Topological vector spaces 1 (Springer-Verlag, Berlin, Heidelberg, New York, 1969).

[10] D.G. Larman and R. Phelps, 'Gateaux differentiabilty of convex functions on Banach spaces', J. London Math. Soc (2) 20 (1979), 115-127.

[11] S. Mazur, 'Über konvexe Mengen in linearen normierten Räumen', Studia Math 4 (1933), 70-84.

[12] Namioka and R. Phelps, 'Banach spaces which are Asplund spaces', Duke Math. J. 42 (1975), 735-750.

[13] R. Phelps, Convex Functions, Monotone Operators and Differentiability: Lecture Notes in Mathematics 1364 (Springer-Verlag, Berlin, Heidelberg, New York, 1989).

[14] A.P. Robertson and W. Robertson, Topological Vector Spaces (Cambridge University Press, Cambridge, 1966).

[15] B. Sharp, Ph.D. Thesis (University of Newcastle, NSW Australia, 1989).

[16] S. Yamamuro, Differential calculus in topological linear spaces: Lecture Notes in Mathematics 374 (Springer-Verlag, Berlin, Heidelberg, New York, 1974). 